\title{
Ueber einen intermediären Aether des Glycols;
}

$$
\text { von A. V. Lourenço *). }
$$

Bromäthylen und Glycol wirken, wenn 4 Tage lang in einem zugeschmolzenen Kolben auf $120^{\circ}$ erhitzt, auf einander ein; es bilden sich dabei Wasser, Bromwasserstoffäther des Glycols, und eine Flüssigkeit, welche ölartig ist wie Glycerin, süfs schmeckt, gegen $245^{\circ}$ siedet, und in Wasser, Alkohol und Aether löslich ist. Die Zusammensetzung der letzteren Flüssigkeil ergab sich aus folgenden Analysen, die mit den Präparaten von verschiedenen Darstellungen (die Siedepunkte lagen zwischen 240 und $250^{\circ}$ ) angestellt wurden :

\begin{tabular}{lccccccc} 
& \multicolumn{4}{c}{} & & & berechnet \\
\cline { 2 - 6 } Kohlenstoff & 44,83 & $\mathbf{4 5 , 1 8}$ & $\mathbf{4 5 , 1 8}$ & $\mathbf{4 5 , 4 3}$ & & $\mathrm{G}_{4}$ & $\mathbf{4 5 , 2 8}$ \\
Wasserstoff & $\mathbf{9 , 5 2}$ & $\mathbf{9 , 4 7}$ & $\mathbf{9 , 6 1}$ & $\mathbf{9 , 6 6}$ & & $\mathrm{H}_{10}$ & $\mathbf{9 , 4 3}$ \\
Sauerstoff & - & - & - & - & & $\Theta_{3}$ & $\mathbf{4 5 , 2 9 .}$
\end{tabular}

Die hiernach sich ergebende Formel $\left.\mathrm{G}_{4} \mathrm{H}_{10} \theta_{3}=\underset{\mathrm{G}_{2}}{\mathrm{G}_{2} \mathrm{H}_{4}} \mathrm{H}_{4}\right\} \theta_{3}$ findet ihre Bestätigung in der Dampfdichte; dieselbe wurde bei $311^{\circ}=3,78$ gefunden, während sie sich $=3,66$ berechnet.

Man kann diese Verbindung als einen intermediären Aether des Glycols betrachten und die Bildung derselben erklären gemäls der Gleichung :

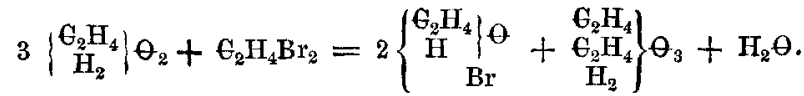
Glycol $\begin{gathered}\text { Brom- } \\ \text { athylen }\end{gathered} \quad \begin{gathered}\text { Bromw.-Aether Intermed. } \\ \text { des Glycols Aether. }\end{gathered}$

*) Compt. rend. XLIX, 619. 
254 Lourenço, über einen intermed. Aether d. Glycols.

Dieser Aether steht zu dem Glycol in einer ähnlichen Beziehung, wie die s. g. Nordhäuser Schwefelsäure zu dem s. g. Schwefelsäurehydrat :

$$
\begin{aligned}
& \left.\left.\underset{\mathrm{G}_{2} \mathrm{H}_{4}}{\mathrm{H}_{2}}\right\} \Theta_{2} \quad \mathrm{~B}_{2} \theta_{2}\right\} \Theta_{2} \\
& \text { Glycol } \\
& \left.\begin{array}{c}
\mathrm{G}_{2} \mathrm{H}_{4} \\
\mathrm{G}_{2} \mathrm{H}_{4} \\
\mathrm{H}_{2}
\end{array}\right\} \theta_{3} \\
& \left.\begin{array}{c}
\mathbb{s}_{2} \theta_{2} \\
\mathbb{s}_{2} \theta_{2} \\
\mathrm{H}_{2}
\end{array}\right\} \theta_{3}
\end{aligned}
$$

Jacquelain hat mit Nordhäuser Schwefelsäure ein krystallisirtes Kalisalz erhalten, dessen Zusammensetzung $\left.\begin{array}{c}\boldsymbol{S}_{2} \theta_{2} \\ \mathbf{S}_{2} \theta_{2} \\ \mathbf{K}_{2}\end{array}\right\} \theta_{3}$ es aufser Zweifel setzt, dafs jene Säure eine wahre chemische Verbindung und nicht eine blofse Mischung ist.

Diese Beispiele zeigen, dafs es unter den Verbindungen zweiatomiger Alkohol- oder Säureradicale eine intermediäre Reihe giebt, die sich bildet, indem zwei Mol. des Alkohols oder der Säure unter Elimination von 1 Mol. Wasser zu einem einzigen zusammentreten; z. B. :

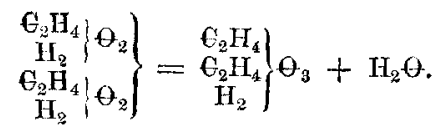

Wenn man der Analogie nach auch auf andere Reihen schliefsen darf, so lälst sich voraussehen, dafs für die dreialomigen Verbindungen, das Glycerin z. B., zwei intermediäre Reihen existiren, welche repräsentirt sind durch die Formeln :

$$
\left.\left.\begin{array}{c}
\mathrm{G}_{3} \mathrm{H}_{5} \\
\mathrm{G}_{3} \mathrm{H}_{5} \\
\mathrm{H}_{4}
\end{array}\right\} \theta_{5} \quad \text { and } \quad \begin{array}{c}
\mathrm{G}_{3} \mathrm{H}_{5} \\
\mathrm{C}_{3} \mathrm{I}_{5} \\
\mathrm{H}_{2}
\end{array}\right\} \theta_{4}
$$

und welche entstehen, indem zwei Mol. Glycerin unter Elimination von 1 oder von 2 Mol. Wasser zu einem einzigen zusammentreten. 